\title{
FATORES DE RISCO EM INDIVÍDUOS COM HIPERTENSÃO ARTERIAL*
}

\author{
Janete Pessuto** \\ Emília Campos de Carvalho***
}

PESSUTO, J.; CARVALHO, E.C. de. Fatores de risco em indivíduos com hipertensão arterial. Rev.latinoam.enfermagem, Ribeirão Preto, v. 6, n. 1, p. 33-39, janeiro 1998.

Este trabalho teve o objetivo de verificar os hábitos relacionados aos fatores de risco em uma população de portadores de hipertensão arterial. A amostra foi composta por 70 indivíduos, constatando-se que a maioria não faz exercício, não tem atividade de lazer, havendo necessidade de programas educativos para aquisição destes hábitos .Há lacunas de informação, pois os clientes têm como ponto de referência, em sua maioria, a própria experiência, ao invés das orientações que recebem de vários profissionais.

UNITERMOS: fatores de risco, hipertensão arterial, programas educativos

\section{INTRODUÇÃO}

A hipertensão arterial, considerada uma doença crônica, pode ser influenciada pelo grau de participação do indivíduo portador de tal patologia, dependendo de fatores como a aceitação da doença, controle e conhecimento da mesma e aparecimento de complicações. É definida como tendo valores de pressão arterial sistólica $>160 \mathrm{~mm} \mathrm{Hg}$ e diastólica $>95 \mathrm{~mm} \mathrm{Hg}$. A hipertensão limítrofe é aquela com valores sistólicos de 140 a 160 mm Hg e diastólicos de 90 a 95 mm Hg. A normotensão é a pressão arterial sistólica $<140 \mathrm{~mm} \mathrm{Hg}$ e diastólica $<90 \mathrm{~mm} \mathrm{Hg}^{7}$.

Um dos principais fatores de risco para complicações cardiovasculares é a hipertensão arterial, pois atua diretamente na parede das artérias, podendo produzir lesões. Daí a importância do tratamento antihipertensivo na redução da morbidade e mortalidade cardiovasculares, principalmente na prevenção de acidentes vasculares, insuficiência cardíaca e renal ${ }^{8}$.

Em 1980 30,8\% de todos os óbitos foram representados pelas doenças cardiovasculares, sendo a primeira causa de mortalidade no país, na população acima de 20 anos de idade. Cerca de $10 \%$ da população adulta tem sua expectativa de vida diminuída devido à hipertensão arterial ${ }^{1,2,11}$.

Vários estudos mostram que existem alguns fatores, considerados fatores de risco que, associados entre si e a outras condições, favorecem o aparecimento da hipertensão arterial, sendo: idade, sexo, antecedentes familiares, raça, obesidade, estresse, vida sedentária, álcool, tabaco, anticoncepcionais, alimentação rica em sódio e gorduras.

O controle da hipertensão arterial inicia-se com a detecção e observação contínua, não devendo ser diagnosticada com base em uma única medida da pressão arterial. Após sua confirmação, deve ser classificada como hipertensão primária ou secundária, verificação do prejuízo dos órgãos alvos como coração, cérebro e rins e levantamento de outros fatores de risco cardiovasculares ${ }^{5}$.

$\mathrm{O}$ tratamento é baseado em três recursos, sendo: não-farmacológico, farmacológico e adesão do cliente ao tratamento 8,18 .

Este trabalho teve por objetivo identificar os hábitos relacionados aos fatores de risco para hipertensão arterial, em indivíduos com esta patologia.

\section{METODOLOGIA}

Realizou-se uma pesquisa do tipo "Survey", através de entrevista estruturada, com a finalidade de se levantar os hábitos do indivíduo portador de hipertensão arterial, relacionados aos fatores de risco da doença.

\footnotetext{
* Este artigo foi extraído da dissertação de mestrado intitulada "As necessidades básicas afetadas e os fatores de risco de clientes portadores de hipertensão arterial”, apresentada ao programa de pós-graduação em Enfermagem, na área de Enfermagem Fundamental, da Escola de Enfermagem de Ribeirão Preto-USP, 1994

** Professor Assistente do Curso de Enfermagem da Faculdade de Medicina de Botucatu - UNESP

*** Professor Titular da Escola de Enfermagem de Ribeirão Preto da Universidade de São Paulo
} 
A investigação ocorreu no Centro de Hipertensão Arterial (CHA), localizado no ambulatório do Hospital das Clínicas da Faculdade de Medicina de Botucatu Universidade Estadual Paulista - UNESP. Esta unidade atende a indivíduos portadores de hipertensão arterial de toda a região, prestando assistência médico-hospitalar e servindo também como campo de pesquisa a diversos profissionais da área de saúde. É um hospital governamental, autárquico, de grande porte, que está vinculado aos programas do Sistema Unificado de Saúde (SUS).

Para este trabalho foram selecionados 70 indivíduos, por ordem de chegada, antes de serem atendidos, para que pudessem ser avaliados no mesmo dia em que se dirigiram ao serviço para receberem atendimento agendado anteriormente.

A amostra selecionada foi formada por indivíduos portadores de hipertensão arterial, de ambos os sexos, cadastrados no CHA, com idade mínima de 18 anos, conscientes e orientados, para que pudessem responder às perguntas da entrevista, que não tinham participado dos grupos de "Sala de Espera", para que não houvesse interferências nos dados coletados e que concordaram em participar da pesquisa.

Os dados foram analisados através da estatística descritiva, já que a amostra foi homogênea.

\section{APRESENTAÇÃO E ANÁLISE DOS RESULTADOS}

Para fazer uma caracterização da amostra estudada, foram levantados os seguintes dados: idade, sexo, cor, estado civil, profissão e escolaridade. A amostra foi composta em sua maioria, por mulheres entre $40 \mathrm{e}$ 50 anos, de cor branca, que se ocupam de prendas domésticas e que apresentam nível de escolaridade inferior ao segundo grau.

Diversos estudos mostram resultados de pesquisas onde tais variáveis foram relacionadas à hipertensão arterial.

Tratando-se da variável idade, vários trabalhos a consideram como um fator de risco importante que contribui para o aparecimento da hipertensão arterial, devido à alterações na musculatura lisa e no tecido conjuntivo dos vasos, como conseqüência do processo de envelhecimento ${ }^{4,5,7,14}$.

Associando idade e sexo, a hipertensão arterial ocorre com maior freqüência no sexo masculino, porém, devido às mudanças de hábitos das mulheres, essa freqüência tem diminuído. As mulheres que fumam e fazem uso de anticoncepcional, com mais de 30 anos, são as mais atingidas. No homem ela aparece depois dos 30 anos e na mulher, após a menopausa. Em ambos os sexos, a freqüência da hipertensão cresce com o aumento da idade, sendo que os homens jovens têm pressão arterial mais elevada que as mulheres, porém após a meia idade este quadro se reverte ${ }^{13,16}$.

Em relação à raça, a negra é mais atingida, sendo que a maior incidência de hipertensão arterial na raça negra ocorre na faixa etária entre 35 a 44 anos ${ }^{4,5,7,15}$.

Tratando-se da profissão, alguns estudos relacionam a profissão/ocupação com a elevação da pressão arterial, sendo que os índices mais baixos de pressão arterial ocorrem no grupo socialmente mais privilegiado e os que nunca trabalharam ocupam uma posição intermediária em relação à prevalência de hipertensão ${ }^{6}$.

Quanto à variável nível de escolaridade, ficou demonstrado que há uma tendência na queda da média da pressão arterial sistólica e da proporção da hipertensão arterial, conforme o grau de educação aumenta. Talvez isso ocorra devido à influência de outros fatores, como a ocupação e fatores de ordem social. Há uma menor prevalência da doença com o aumento do nível de escolaridade, sendo este dado relevante, já que irá interferir diretamente na assimilação das orientações necessárias ao tratamento ${ }^{6,12}$.

A seguir serão apresentados os dados referentes a alguns fatores de risco da hipertensão arterial, sendo: execução ou não de atividade física, atividade de lazer, utilização de sal e gordura na alimentação, utilização de álcool e tabaco. Serão apresentados também os dados relativos às respostas dos indivíduos sobre a sua opinião em relação à interferência destes fatores na pressão arterial e como eles chegaram a essa conclusão.

O exercício contribui na redução da obesidade e para a prevenção de doenças coronárias. Também auxilia na preservação da independência de pessoas idosas, melhorando o funcionamento do organismo, reforçando o coração, músculos, pulmões, ossos e articulação. A atividade física realizada regularmente melhora a condição física e a saúde do coração, devendo o exercício ser realizado, no mínimo, três vezes por semana, com duração de pelo menos vinte minutos, ser uma atividade regular, pois quando a mesma é interrompida a condição física deteriora-se rapidamente ${ }^{10}$.

No que diz respeito a execução de atividade física, dos indivíduos que participaram da amostra, $62,8 \%$ não fazem exercício, como pode ser observado na Tabela 1. 
Tabela 1- Distribuição dos clientes do C.H.A., de acordo com a execução de atividade física

TIPO DE EXERCÍCIO FREQÜÊNCIA PERCENTUAL

\begin{tabular}{ccc}
\hline não faz exercício & 44 & 62,8 \\
caminhada & 20 & 28,6 \\
outros & 3 & 4,3 \\
ginástica & 2 & 2,9 \\
caminhada e ginástica & 1 & 1,4 \\
\hline TOTAL & 70 & 100,0 \\
\hline
\end{tabular}

Dentre os indivíduos que realizam atividade física, $28,6 \%$ fazem caminhada, sendo que a periodicidade maior é a diária $(21,7 \%)$, seguida da categoria sem periodicidade $(4,3 \%)$ e depois, das categorias duas e três vezes por semana (2,9\% cada).

Quanto à interterência do exercício na pressão arterial, os dados estão demonstrados na Tabela 2.

Tabela 2- Distribuição dos clientes do C.H.A., de acordo com a opinião dos mesmos, sobre a interferência do exercício na pressão arterial

\section{INTERFERÊNCIA FREQÜÊHCIA PERCENTUAL}

\begin{tabular}{lcc}
\hline faz bem & 25 & 35,7 \\
nãosabe & 20 & 28,6 \\
faz mal & 16 & 22,8 \\
não interfere & 9 & 12,9 \\
\hline \multirow{2}{*}{ TOTAL } & 70 & 100,0 \\
\hline
\end{tabular}

Quanto à conclusão de tais respostas, 41,5\% responderam basearem-se na experiência própria, 17,1\% receberam orientação médica, 4,3\% fizeram leituras sobre o assunto, 2,9\% não souberam responder, $1,4 \%$ receberam orientação em grupo, $28,5 \%$ não responderam e 4,3\% deram diversas respostas.

Buscando identificar a execução de atividades de lazer e estratégias de redução de stress, os clientes do C.H.A. foram questionados quanto a sua opinião sobre o seu estado emocional mais freqüente, se eles se vêem como pessoas nervosas ou calmas. A maioria respondeu que se acha nervosa (82,9\%), sendo que $52,9 \%$ delas não desenvolvem atividades de lazer que poderiam contribuir para amenizar tal comportamento. Mecanismos mal adaptados , como por exemplo, manter a raiva reprimida, são fatores que contribuem para a elevação da pressão sangüínea diastólica, mais do que a raça, sendo que o relaxamento pode contribuir para a redução dos efeitos estressantes ${ }^{7}$. Quanto à interferência do nervosismo na pressão arterial, $90,0 \%$ responderam que há interferência, $8,6 \%$ não sabem e $1,4 \%$ acreditam que não interfere.
Concluíram sobre tal assunto através da experiência própria $61,4 \%$ dos indivíduos, $11,3 \%$ não sabem, $5,8 \%$ devido à orientação médica, $4,3 \%$ receberam orientação em grupo e o restante forneceu outras respostas.

Outro fator de risco estudado foi a quantidade de sal na dieta. Questionados sobre a alimentação, grande parte dos clientes $(74,3 \%)$ respondeu que sua comida é preparada com pouco sal e apenas um indivíduo respondeu que utilizava bastante sal. Porém, observa-se que, apesar de a maioria ter dado tal resposta, fica a dúvida em relação à quantidade ideal de consumo de sal, pois conceitos de pouco, muito e médio são variáveis de pessoa para pessoa, por não possibilitarem uma medida real. Deve haver um estudo no sentido de se encontrar uma medida caseira que expresse realmente os valores permitidos para o consumo.

Quanto ao uso de sal, 85,7\% acreditam que o sal interfere na pressão arterial, 8,6\% não sabem, 4,3\% acham que não há interferência e 1,4\% não responderam. Quanto à conclusão desta questão, 40,0\% concluíram por experiência própria, $25,6 \%$ por orientação médica, $5,8 \%$ receberam orientação em grupo e os demais deram outras respostas.

Tratando-se ainda de alimentação, os clientes do C.H.A. foram abordados sobre a ingestão de gorduras, sendo que $47,1 \%$ responderam que não gostam desse tipo de alimento; $7,1 \%$ responderam que gostam, mas não comem; os demais $(45,8 \%)$ referiram consumir alimentos variados que são ricos em gorduras, sendo: carne gorda $(8,6 \%)$, fritura $(8,6 \%)$, os dois tipos citados juntos $(11,4 \%)$, banha de porco $(4,3 \%)$, presunto associado aos itens já mencionados $(1,4 \%$ de cada item). Quanto à periodicidade, não há um período freqüente para os que utilizam tais alimentos.

A maioria dos indivíduos respondeu não fazer uso de gorduras em sua alimentação, porém, quando indagados sobre o consumo de carne, leite e ovos, que são alimentos ricos em gorduras, mais de $70 \%$ afirmaram que utilizam tais alimentos com freqüência. De acordo com alguns estudos, a ingestão de gorduras em indivíduos portadores de hipertensão arterial deve ser reduzida, pois há aumento das complicações, como doenças coronárias, e também pode haver obesidade que, por si só, eleva os valores tensionais ${ }^{9}$. A maioria dos clientes $(72,8 \%)$ referiu que as gorduras interferem na hipertensão arterial; 14,3\% não sabiam se haviam relações entre tais fatores e 12,9\% mencionaram não haver interferência.

O álcool é outro fator de risco, comentado na literatura, que contribui para o agravamento da patologia. O aumento das taxas de álcool no sangue eleva a pressão arterial lenta e progressivamente, na proporção de $2 \mathrm{~mm}$ $\mathrm{Hg}$ para cada $30 \mathrm{ml}$ de álcool etílico ingeridos diariamente, sendo que quando suspenso, as cifras revertem ${ }^{9}$. 
Outro dado interessante é que quando há redução no consumo de álcool, ocorre também redução no abandono do tratamento farmacológico ${ }^{15}$.

Neste trabalho, $72,9 \%$ dos clientes disseram que não bebem nenhum tipo de bebida alcóolica e entre os que afirmaram beber, $17 \%$ bebem cerveja, sem uma periodicidade específica. Pode-se observar tais resultados na Tabela 3.

Tabela 3 - Distribuição dos clientes do C.H.A., de acordo com a utilização de bebida alcoólica

\section{TIPO DE BEBIDA FREQÜÊNCIA PERCENTUAL}

\begin{tabular}{ccc}
\hline não bebe & 51 & 72,9 \\
cerveja & 12 & 17,1 \\
vinho & 2 & 2,9 \\
pinga e cerveja & 2 & 2,9 \\
pinga & 1 & 1,4 \\
cerveja e vinho & 1 & 1,4 \\
wisk & 1 & 1,4 \\
\hline TOTAL & 70 & 100,0 \\
\hline
\end{tabular}

Em relação à quantidade de bebida ingerida, houve uma variação grande, já que dependeu do tipo de bebida utilizada. Porém, a maioria referiu ingerir pequenas quantidades, sendo que para quem toma vinho, um cálice cerca de três vezes por semana; para quem toma cerveja, uma garrafa aos finais de semana, na maioria dos casos.

Sobre a interferência do álcool na pressão arterial, $74,3 \%$ responderam que há interferência e $25,7 \%$ não souberam responder. Chegaram a esta conclusão: $22,9 \%$ por experiência própria, $17,1 \%$ não sabem, 10,0\% receberam orientação médica, $4,3 \%$ receberam orientação em grupo, $15,7 \%$ deram outras respostas e $25,7 \%$ não responderam.

Quanto à utilização de tabaco, 25,8\% dos indivíduos declararam fumar. Grande parte dos que fumam disse consumir até dez cigarros por dia, como pode ser observado na Tabela 4.

Tabela 4- Distribuição dos clientes do C.H.A., de acordo com a utilização de tabaco

\begin{tabular}{ccc}
\hline UTLIZAÇÃO & FREQÜENHCIA & PERCENTUAL \\
DE TABACO & & \\
\hline não utiliza & 52 & 74,3 \\
10 cigarros/dia & 9 & 12,8 \\
5 cigarros/dia & 5 & 7,1 \\
20 cigarros/dia & 2 & 2,9 \\
outras quantidades & 2 & 2,9 \\
\hline TOTAL & 70 & 100,0 \\
\hline
\end{tabular}

A nicotina é prejudicial ao organismo, pois promove a liberação de catecolaminas, que aumentam a freqüência cardíaca, a pressão arterial e a resistência periférica. Aumenta também a capacidade orgânica em formar coágulos e diminui sua função de destruí-los. Há redução de oxigênio nos glóbulos vermelhos em cerca de 15 a $20 \%$, pois o monóxido de carbono que resulta da queima do fumo e do papel,se liga à hemoglobina. Este último também lesa a parede interna dos vasos, propiciando a deposição de gorduras ${ }^{6,17}$.

Dos indivíduos entrevistados, 67,2\% responderam que o tabaco interfere na pressão arterial, $27,1 \%$ não sabem $1,4 \%$ responderam que não há interferência e 4,3\% não deram tal informação. Chegaram a essa conclusão através da experiência própria $27,5 \%$ dos indivíduos, $17,6 \%$ receberam orientação médica, $15,7 \%$ não souberam responder, 9,8\% concluíram baseados em leituras e $29,5 \%$ deram outras respostas.

Quanto à interferência dos fatores de risco já mencionados, conforme a opinião dos clientes, a atividade física e a atividade de lazer e recreação não são realizadas. Há necessidade de uma intervenção educativa mais esclarecedora, já que a execução de tais atividades é recomendada para o tratamento da hipertensão arterial e para a prevenção de complicações.

A partir das respostas dadas ao instrumento de coleta de dados, fica evidente que a experiência própria é um elemento importante para o cliente concluir a respeito dos efeitos de determinados procedimentos sobre a pressão arterial. Para a realização de exercícios, $40 \%$ dos indivíduos se basearam em experiências próprias; para a questão sobre o nervosismo, esta categoria subiu para $60 \%$; quanto ao uso do sal $40 \%$; uso de gorduras 41,4\%; utilização de álcool 22,9\%; uso de tabaco $20 \%$. Quando a categoria "outras" foi citada, em muitos casos eles comentavam outras experiências pessoais, como o problema de um vizinho, de um parente.

A orientação médica ficou em segundo lugar, com uma porcentagem bem inferior, pois as respostas foram muito diversificadas quando elas saíam da categoria da experiência de cada um.

A enfermeira não foi citada em momento algum, apesar do trabalho que desenvolvia com esses clientes, tanto nos grupos de orientação, como nos atendimentos após a consulta médica (pós-consulta de enfermagem).

De acordo com a literatura pesquisada, sabe-se que o objetivo de se acompanhar o portador de hipertensão arterial é controlar sua pressão e os fatores de risco ${ }^{1}$. Entretanto, não bastam medidas de orientação, mas sim devem haver estratégias que auxiliem o indivíduo na mudança para comportamentos que contribuam com o controle da doença. As medidas educacionais devem ser contínuas, já que várias são as causas da não adesão ao tratamento, sendo uma delas, a falta de motivação, 
podendo estar associada principalmente aos fatores ambientais $^{3}$.

Para que os clientes sintam-se motivados a participarem ativamente do tratamento da hipertensão arterial, acredita-se que o caminho seja a educação e a estimulação para a mudança de atitudes diante da patologia. Deve haver também, um maior envolvimento dos profissionais que participam dos programas de atendimento, oferecendo suporte social adequado, através de uma relação social mais próxima, na qual tenha afetividade, comunicação e visão do cliente como único, levando-se em consideração seus problemas e sua história de vida.

\section{CONCLUSÃO}

Considerando o objetivo do presente estudo de identificar os hábitos relacionados aos fatores de risco para hipertensão arterial, em indivíduos com esta patologia, podemos concluir para a presente amostra, que:

- 35,7\% dos sujeitos consideram que o exercício físico interfere positivamente na pressão arterial; $28,6 \%$ não sabem se há relação entre tais fatores; $22,8 \%$ referem que a interferência é maléfica e $12,9 \%$ destes mencionam não haver interferência. Contudo, $62,8 \%$ dos sujeitos não fazem exercício; dentre os que fazem atividade física, a maioria $(28,6 \%)$ realiza a caminhada diária.

- 90,0\% responderam que há interferência do nervosismo na pressão arterial. A maioria dos sujeitos considera-se nervosa $(82,9 \%)$, sendo que $52,9 \%$ deles não desenvolvem atividades que contribuiria para reduzir tal situação.

- 85,7\% acreditam que o sal interfere na pressão arterial; $74,3 \%$ relatam ingerir pouco sal; não foi possível quantificar o sal utilizado.

- 72,8\% julgam que há interferência da gordura na pressão arterial; $14,3 \%$ não sabem e $12,9 \%$ consideram que não interfere. Destaca-se que, $47,1 \%$ mencionaram não gostar deste tipo de alimento, enquanto 45,8\% afirmaram consumir alimentos ricos em gorduras.
Contudo, ao serem indagados sobre consumo de carnes, leite e ovos, mais de $70 \%$ dos sujeitos confirmaram consumir tais alimentos freqüentemente.

- 74,3\% responderam que há interferência do álcool sobre a pressão arterial. Dos sujeitos $72,9 \%$ mencionaram que não bebem; $17 \%$ bebem, preferencialmente cerveja, de forma esporádica.

- 67,2\% declararam que o fumo interfere na pressão arterial. A maioria (74,3\%) mencionou que não fuma; dos $25,8 \%$ que afirmaram que fumam, grande parte consome até 10 cigarros/dia.

- A maioria das opiniões sobre as relações entre os fatores de risco e a hipertensão arterial foi concluída, segundo os sujeitos, predominantemente por experiências próprias, seguindo-se a orientação médica. O profissional enfermeiro não foi citado, embora no serviço que atende estes sujeitos haja atuação de enfermeiro, quer nos grupos de orientação quer no atendimento pós consulta.

\section{CONSIDERAÇÕES FINAIS}

De acordo com os dados levantados neste trabalho, entende-se que há necessidade de organizar um atendimento a esses clientes, no sentido de fortalecer a importância de mudanças de comportamentos, já que foram levantados aspectos falhos quanto à atividade física e de lazer, uso de tabaco, assim como dados questionáveis em relação à alimentação.

Outro ponto é com relação aos profissionais que os atendem, visto que a enfermeira não é reconhecida dentro da equipe, por parte do cliente. É importante que haja a divulgação do trabalho de todos os profissionais, e que o cliente saiba valorizá-los.

Acredita-se que a educação dos indivíduos portadores de hipertensão arterial seja o melhor caminho para o alcance de tais objetivos, não sendo apenas uma transmissão de conteúdos referentes à patologia e ao tratamento, mas sim que se promova a adaptação dos clientes ao tratamento da hipertensão arterial. Para se chegar a essa adaptação, é preciso que os indivíduos estejam motivados para que tais mudanças ocorram e, também, para que assimilem os conhecimentos que poderão melhorar a qualidade de vida dos mesmos.

\section{RISK FACTORS TO PATIENTS WITH ARTERIAL HYPERTENSION}

The aim of this research was to verify the habits related to the risk factors of one population with arterial hypertension. Seventy individuals were interviewed. Authors found that the majority do not practice any kind of physical exercise, they do not have leisure activities and need education programs to aquire these habits. There are gaps in information, because they have their own experience as a reference, in spite of the orientations they received from several professionals. 


\section{FACTORES DE RIESGO EN INDIVIDUOS CON HIPERTENSIÓN ARTERIAL}

Este trabajo tuvo como objetivo verificar los hábitos relacionados con los factores de riesgo en una población de portadores de hipertensión arterial. Fueron entrevistados setenta individuos, y se constató que la gran mayoría no práctican ejercicios físicos y no tienen actividades de recreación, existiendo la necesidad de programas educativos para la aquisición de estes hábitos. Hay lagunas de información, pues la mayoría del clientes tienen como punto de referencia sus propias experiencias y no las orientaciones recibidas de varios profesionales.

TÉRMINOS CLAVES: factores de riesgo, hipertensión arterial, programas educativos

\section{REFERÊNCIAS BIBLIOGRÁFICAS}

01. BRASIL. Ministério da Saúde. Secretaria Nacional de Programas Especiais de Saúde. Divisão Nacional de Doenças Crônico-Degenerativas. Guia para controle da hipertensão arterial. Brasília: Centro de Documentação do Ministério da Saúde,1983. 26 p.

02. BRASIL. Ministério da Saúde. Secretaria Nacional de Programas Especiais de Saúde. Divisão Regional de Doenças Crônico-Degenerativas. Programa Nacional de Educação e Controle da Hipertensão Arterial. Brasília , 1988. 48 p.

03. CAMPOS, E. P.; LEITE, I. de S. C. Fatores terapêuticos de um programa continuado e integrado de atendimento ao hipertenso. Rev. Prática Médica, v. 101, p. 9-14, 1990.

04. FRANCISCHETTI, E. A. et al. Hipertensão arterial leve: o que além das cifras? Arq.Bras.Cardiol., v. 52, p. 349-65, 1989.

05. JOINT NATIONAL COMMITTEE ON DETECTION, EVALUATION AND TREATMENT OF HIGH BLOOD PRESSURE. The fifth report. Arch. Intern.Med., v. 153, p. 154-83, 1993.

06. KLEIN, C. H., ARAUJO, J. W. G. de. Fumo, bebida alcóolica, migração, instrução, ocupação, agregação familiar e pressão arterial em Volta Redonda, Rio de Janeiro. Cad. Saúde Pública, v. 1, p. 160-76, 1985.

07. KOCHAR, M. S.; WOODS, K. D. Controle da hipertensão: para enfermeiras e demais profissionais de saúde. 2. ed. São Paulo: Andrei, 1990. 317 p.

08. MARANHÃO, M. F. de C.; RAMIRES, J. A. F. Aspectos atuais do tratamento da hipertensão arterial. Arq. Bras. Cardiol., v. 51, p. 99-105, 1988.
09. MION JÚNIOR, D. Hipertensão: aspectos práticos. São Paulo: Sociedade Brasileira de Cardiologia, Departamento de Hipertensão Arterial, 1988.16p.

10. MORRIS, J.; COLliNS, M. Saúde graças ao exercício-uma lei da natureza. Saúde Mundo, p. 6-7, 1992.

11. ORGANIZAÇÃO PANAMERICANA DE SAÚDE. A hipertensão arterial como problema de saúde comunitária. Manual de normas operacionais para um programa de controle nos diferentes níveis de atenção, 1986. p. 1-68.

12. PIERIN, A. M. G. Orientação sistematizada do paciente hipertenso submetido a tratamento ambulatorial. Rev. Esc. Enfermagem USP, v. 20, p. 193-205, 1986.

13. RIBEIRO, A. B. Atualização em hipertensão arterial: clínica, diagnóstico e terapêutica. São Paulo: Atheneu, 1996. $231 \mathrm{p}$.

14. ROCCELLA, E. J. et al. Considerações epidemiológicas em definir a hipertensão. Clín. Méd. Am. Norte, v.5, p. 811-29, 1987.

15. SCHMIEDER, R. E. et al. Riesgos en el desarrollo de hipertensión arterial. Medicina, Buenos Aires, v. 48, p. 303-10, 1988.

16. SILVA, H. B.; CASTRO, P. J. B. Hipertensão arterial. In: MARCONDES, M.,SUSTOVICH, D. R.; RAMOS, O. L. Clínica médica: propedêutica e fisiopatologia. $2^{\mathrm{a}}$ ed. Rio de Janeiro: Guanabara Koogan, 1979. cap. 16, p. 911-3.

17. SILVA, M. A. D. da. Guerra contra o fumo: opção pela vida. São Paulo: Fundo de Aperfeiçoamento e Pesquisa em Cardiologia, s.d. 8 p.

18. VENTURA, J .E. Principios del tratamiento de la hipertensión arterial essencial. Rev.Méd.Urug., v. 2, p. 40-50, 1986. 


\section{FORMULÁRIO $\mathbf{N}^{\circ}$}

\section{I- DADOS PESSOAIS:}

01. Data de nascimento: anos

02. Sexo:

03. Raça:

04. Qual o seu estado civil?

05. Qual a sua profissão (ou ocupação)?

06. Qual o nível de escolaridade?

\section{II- DADOS RELATIVOS AOS FATORES DE RISCO:}

01. Faz uso de anticoncepcional?

02. Faz exercícios? Qual a freqüência e o tipo?

03. Na sua opinião, os exercícios interferem na pressão arterial?

04. Como chegou a essa conclusão?

05 . Você se acha uma pessoa calma ou nervosa?

06. Como se diverte?

07. Na sua opinião, o nervosismo interfere na pressão arterial?

08. Como chegou a essa conclusão?

09. Como é preparada sua comida, em relação à quantidade de sal?

10. Na sua opinião, o sal interfere na pressão arterial?

11. Como chegou a essa conclusão?

12. Gosta de alimentos que contêm gordura?

13. Na sua opinião, os alimentos gordurosos interferem na pressão arterial?

14. Como chegou a essa conclusão?

15. Você bebe algum tipo de bebida alcoólica? Qual a quantidade e o tipo?

16. Na sua opinião, o álcool interfere na pressão arterial?

17. Como chegou a essa conclusão?

18. Você fuma? Qual a quantidade?

19. Na sua opinião, o tabaco interfere na pressão arterial?

20. Como chegou a essa conclusão? 